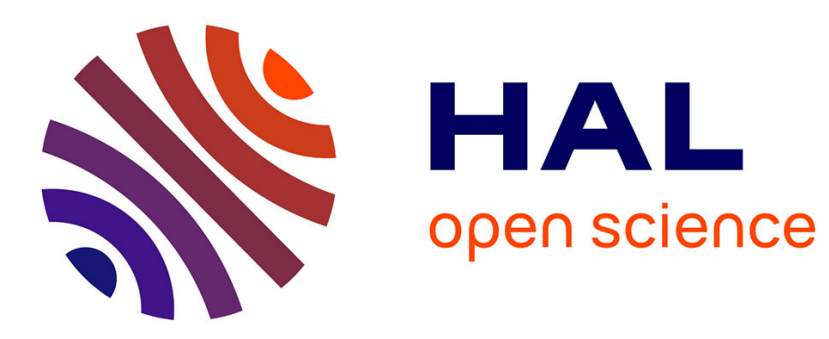

\title{
Nonlinear Analysis of Stripe Textures in Hexagonal Lyotropic Mesophases
}

\author{
P. Oswald, J. Géminard, L. Lejcek, L. Sallen
}

\section{To cite this version:}

P. Oswald, J. Géminard, L. Lejcek, L. Sallen. Nonlinear Analysis of Stripe Textures in Hexagonal Lyotropic Mesophases. Journal de Physique II, 1996, 6 (2), pp.281-303. 10.1051/jp2:1996182 . jpa00248298

\section{HAL Id: jpa-00248298 https://hal.science/jpa-00248298}

Submitted on 1 Jan 1996

HAL is a multi-disciplinary open access archive for the deposit and dissemination of scientific research documents, whether they are published or not. The documents may come from teaching and research institutions in France or abroad, or from public or private research centers.
L'archive ouverte pluridisciplinaire HAL, est destinée au dépôt et à la diffusion de documents scientifiques de niveau recherche, publiés ou non, émanant des établissements d'enseignement et de recherche français ou étrangers, des laboratoires publics ou privés. 


\title{
Nonlinear Analysis of Stripe Textures in Hexagonal Lyotropic Mesophases
}

\author{
P. Oswald (*), J.C. Géminard, L. Lejcek $\left(^{* *}\right)$ and L. Sallen \\ Laboratoire de Physique, École Normale Supérieure de Lyon, 46 Allée d'Italie, \\ 69364 Lyon Cedex 07, France
}

(Received 13 July 1995, revised 20 October 1995, accepted 23 October 1995)

\author{
PACS.61.30.Eb - Experimental determinations of smectic, nematic, cholesteric, \\ and lyotropic structures \\ PACS.62.20.Dc - Elasticity, elastic constants \\ PACS.81.40.Jj - Elasticity and anelasticity
}

\begin{abstract}
Fan-shaped textures of hexagonal mesophases of lyotropic systems are often striated. These striations are due to a thermomechanical undulation of the columns. We measure their wavelength and amplitude in the lyotropic mixture $\mathrm{C}_{12} \mathrm{EO}_{6}$ +water. By comparing these measurements to the theoretical predictions in the strongly nonlinear regime (our model is a generalization of the model of Singer [17] to columnar phases), we find that the penetration length $\lambda=\sqrt{K / B}$ is of the order of $40 \AA$ (where $B$ is the compressibility modulus of the hexagonal array and $K$ the curvature modulus of the columns). This value is in qualitative agreement with that found by using X-rays [11] or the grain-boundary method [10]. In addition, we find that the shear elastic modulus $\mu$ of the hexagonal array is 5 to 10 times smaller than $B$, in agreement with X-ray experiments [16].
\end{abstract}

Résumé. - Les textures en éventail des phases colonnaires hexagonales des systèmes lyotropes sont souvent striées. Ces striations sont dues à une ondulation d'origine thermomécanique des colonnes. Nous avons mesuré leur longueur d'onde et leur amplitude dans le mélange lyotrope $\mathrm{C}_{12} \mathrm{EO}_{6}+$ eau. En comparant ces mesures aux prédictions théoriques dans le régime fortement non linéaire (notre modèle est une généralisation du modèle de Singer [17] aux phases colonnaires), nous avons trouvé que la longueur de pénétration $\lambda=\sqrt{K / B}$ était de l'ordre de $40 \AA$ ( $B$ est le module de compressibilité du réseau hexagonal et $K$ le module de courbure des colonnes). Cette valeur est en accord qualitatif avec celles trouvées par rayons X [11] ou par la méthode du joint de grain [10]. Par ailleurs, nous avons trouvé que le module de cisaillement $\mu$ du réseau hexagonal est environ 5 à 10 fois plus petit que $B$, en accord avec les expériences de rayons $\mathrm{X}[16]$.

${ }^{*}$ ) Author for correspondence (oswald@physique.ens-lyon.fr)

$\left(^{* *}\right)$ Permanent address: Institute of Physics, Czech Academy of Sciences, Na Slovance 2. 18040 Prague

8, Czech Republic. 


\section{Introduction}

Fan-shaped textures of hexagonal mesophases of lyotropic systems are often striated. The hexagonal phase consists of parallel cylinders of surfactant molecules, separated by water, which form a hexagonal array in a plane perpendicular to their axis. These striations are easily seen through the microscope between crossed polarizers and form spontaneously when the phase is cooled down. They occur as a succession of black and white stripes which are perpendicular to the mean direction of the optical axis parallel to the molecular cylinders. These striations have been observed by numerous authors in lyotropic systems [1-3] and are due to an undulation or to a zigzag of the columns. These striations are also visible in columnar mesophases of discotic liquid crystals. They nucleate by suddenly dilating planar samples perpendicularly to the columns [4]. The columns then undulate above some critical thickness variation with a well-defined wavelength. This mechanical instability can be analyzed as in smectics [5] and results from a competition between the compressional energy of the hexagonal array and the curvature energy of the columns. From the measurements of the undulation wavelength as a function of the sample thickness, Gharbia et al. [4] found that the apparent curvature elastic modulus of the columns $K_{\text {app }}$ was anomalously large in discotic liquid crystals, i.e., 6 orders of magnitude larger than in usual liquid crystals like smectics or nematics. They found a similar value by measuring the threshold of column buckling under compression along the columns [6]. A possible explanation for this unusual behavior was given by Prost [7] by assuming that there exists a large density of column entranglements. These defects could make the system stiffer at large scale on condition that they do not move by permeation during the buckling process (that assumes that the frequency is large enough). These defects could also be responsible for the solid-like Rayleigh scattering recently observed in discotic mesophases by Gharbia et al. [8]. It could also explain the presence of an apparent shear modulus $C_{44}$ parallel to the columns which is two orders of magnitude smaller than the compressibility modulus of the hexagonal array [9].

In order to complete these results, we recently measured the curvature modulus $K$ of an isolated column. This measurement was done by estimating the energy of transverse edge dislocations [10]. This purely static measurement showed that, at small scale (within $1 \mu \mathrm{m}$ ), $K$ has an usual value (of the order of $10^{-6}$ dyne as in nematics or smectics), both in thermotropic and lyotropic systems, in agreement with X-ray measurements [11].

In this article, we analyze the mechanisms of formation of the striations which form spontaneously when a planar sample of a lyotropic hexagonal mesophase is slowly grown from its isotropic liquid (for the phase diagram, see [12]). These undulations of the columns are due to thermal effects, i.e. both to a density jump at the phase transition and to a decrease of the lattice parameter when the temperature decreases [13]. A similar explanation was already proposed by Petrov and Durand [14] in lyotropic lamellar systems to explain the spontaneous undulations of the layers when the temperature changes. The main difference with previous buckling experiments in discotic liquid crystals is that, now, the dilation is two-dimensional, in the plane perpendicular to the columns. Also, it is imposed very slowly so that the system remains very close to its mechanical equilibrium at zero-frequency. Our purpose is to show that the typical wavelength of thermal undulations which are observed in the non linear regime (i.e. when columns form zigzags, far from the critical threshold) are compatible with our previous measurements of $K$ in lyotropic systems, without resorting to some abnormal elasticity.

The plan of the article is as follows. First, we present experimental measurements. Second, we show how to calculate the wavelength, first in the linear regime (threshold) and then in the nonlinear regime when zigzags form. Finally, we discuss our results. 


\section{Experimental}

The system chosen is the binary mixture $\mathrm{C}_{12} \mathrm{EO}_{6}$ + water at the azeotropic concentration ( $49.6 \%$ by weight of polyoxyethylene glycol) $[12,13]$. Samples are prepared between two glass plates and are filled by capillarity in a moist atmosphere. They are sealed with an epoxy glue (Epotecny Luxtrak LCR 0208). Their thicknesses range between 40 and $180 \mu \mathrm{m}$. Below $40 \mu \mathrm{m}$, the samples are very long to fill by capillarity (10 $\mathrm{mn}$ or more) and their water concentration can change. On the other hand, they are very difficult to orient when they are thicker than $200 \mu \mathrm{m}$. The samples are slowly grown in directional solidification [15] from their isotropic micellar phase. The maximum usable pulling velocity is close to $5 \mu \mathrm{m} / \mathrm{s}$ at the azeotropic point. Above this velocity, nucleation occurs in front of the interface and samples disorient. Below this velocity, it is possible to obtain large planar monodomains with all the columns oriented in a single direction (within $1^{\circ}$ ) parallel to the glass plates. The surface area of the monodomains can be as large as $1 \mathrm{~cm}^{2}$. Nevertheless we performed most of our measurements on smaller monodomains (of a few $\mathrm{mm}^{2}$ usually). We also checked that our results were independent of the size of the domain chosen. In practice, the monodomains are always striated independently of the column orientation with respect to the growth front. The striations occur immediately behind the front and their wavelength does not depend on growth velocity and on their orientation with respect to the front. Consequently, they are not due to some interfacial instability. Their optical contrast between crossed polarizers increases when the temperature decreases, so we did our observations a few degrees below the transition temperature. In these conditions, striations are similar, but much more contrasted (Fig. 1). In Figure 2, we plotted the optical contrast of the striations between crossed polarizers as a function of angle $\varphi$ between the polarizer and the average direction of the columns (perpendicular to the striations) for a sample of thickness $d=150 \mu \mathrm{m}$. The optical contrast of the stripes between crossed polarizers is defined to be:

$$
C_{\perp}=\frac{I_{\text {zag }}-I_{\text {zig }}}{I_{\text {zag }}+I_{\text {zig }}}
$$

This quantity was measured in monochromatic light at $\bar{\lambda}=542 \mathrm{~nm}$ with a Leitz microscope equipped with a computer-assisted imaging system composed of a S.I.T. Hamamatsu Videocamera and a Quadra-700 Apple Macintosh computer. Measurements were done after removing the condensor of the microscope in order to obtain parallel rays. Figure 1 shows that the contrast changes sign when $\varphi$ changes sign while Figure 2 shows that the contrast passes through a maximum for an angle $\varphi_{\max }$ of a few degrees. That means that the columns undulate in a plane parallel to the glass plates. In appendix 1 , we have calculated $C_{\perp}$ as a function of the maximum tilt angle $\theta$ of the columns with respect to their average direction. This calculation gives for small angles $\varphi$ and $\theta$ :

$$
C_{\perp}=\frac{a \theta \varphi}{b \varphi^{2}+c \theta^{2}}
$$

where the coefficients $a, b$ and $c$ depend on the way the columns rotate within the sample. We shall see in Section 4 that the columns remain parallel to the glass plates and form a zigzag. The angle $\bar{\theta}(x)$ that they make with their average direction can be taken in the form:

$$
\vec{\theta}(x)= \begin{cases}\theta \sin \left(\frac{\pi}{\chi} \frac{x}{d}\right) & 0<x<d \frac{\chi}{2} \\ \theta & d \frac{\chi}{2}<x<d\left(1-\frac{\chi}{2}\right) \\ \theta \sin \left[\frac{\pi}{\chi}\left(1-\frac{x}{d}\right)\right] & d\left(1-\frac{\chi}{2}\right)<x<d\end{cases}
$$



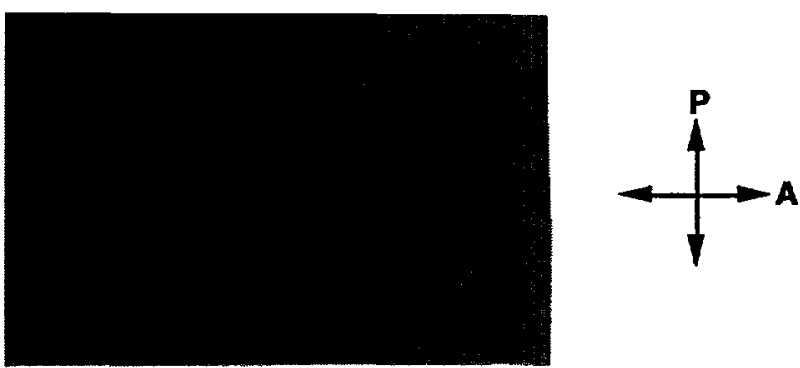

(a)
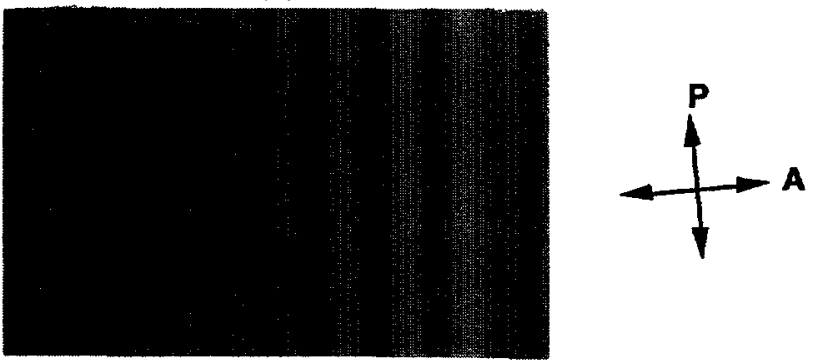

(b)
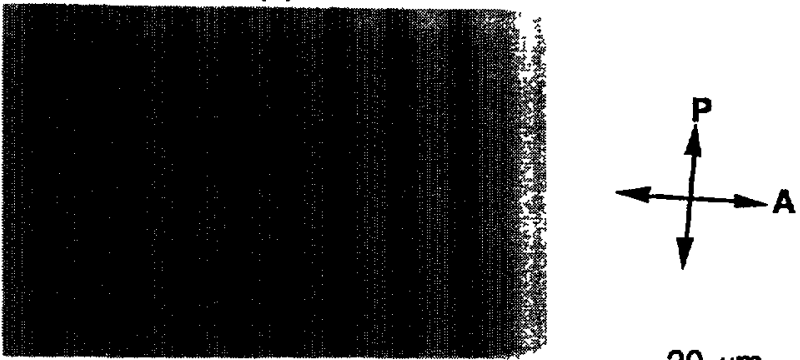

(c)

Fig. 1. - Striations observed in crossed polarizers illumination for different values of the angle $\varphi$ between the polarizer and the mean direction of the molecular columns: a) at $\varphi=0$, the stripes are almost invisible and their contrast vanishes; b) at $\varphi=5^{\circ}$, their contrast is maximum c) at $\varphi=-5^{\circ}$, their contrast is still maximum but is inverted in comparison with the previous case.

In this expression, $\chi$ is a dimensionless constant ranging between 0 and 1 and $d$ is the sample thickness. Coefficients $a, b$ and $c$ can be analytically calculated in the two limiting cases $\chi=0$ and $\chi=1$. This calculation gives (see appendix $A$ ):

$$
a=2, \quad b=c=1 \quad \text { for } x=0
$$

and

$$
\begin{aligned}
a & =\pi \Delta \Phi \sin (\Delta \Phi)\left[\pi^{2}-(\Delta \Phi)^{2}\right] \\
b & =\left[\pi^{2}-(\Delta \Phi)^{2}\right]^{2} \sin ^{2}\left(\frac{\Delta \Phi}{2}\right) \quad \text { for } \chi=1 \\
c & =\pi^{2}(\Delta \Phi)^{2} \cos ^{2}\left(\frac{\Delta \Phi}{2}\right)
\end{aligned}
$$




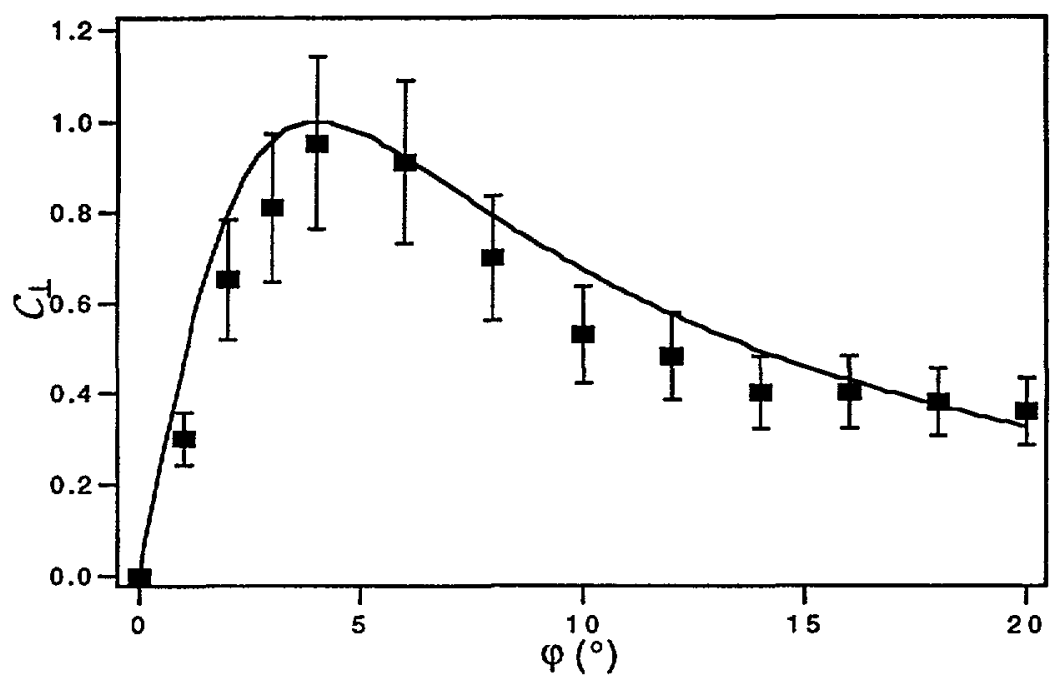

Fig. 2. - Optical contrast $\mathcal{C}_{\perp}$ of the striations at $\bar{\lambda}=542 \mathrm{~nm}$ as a function of angle $\varphi$. The solid line is the best fit to theoretical law (2) (equivalent to Eq. (A.11) of Appendix A). It yields $\theta \approx 4^{\circ}$.

where $\Delta \Phi=\frac{2 \pi d \Delta n}{\bar{\lambda}}$ is the phase shift between the ordinary and the extraordinary rays, $\Delta n$ the birefringence (of the order of $1.5 \times 10^{-3}$, see appendix $B$ ) and $\bar{\lambda}$ the light wavelength. The contrast is maximum and equal to 1 for

$$
\begin{aligned}
& \varphi=\varphi_{\max }=\theta \quad \text { when } \quad \chi=0 \\
& \varphi=\varphi_{\max }=\frac{\pi \theta \Delta \Phi}{\pi^{2}-(\Delta \Phi)^{2}} \cot \left(\frac{\Delta \Phi}{2}\right) \quad \text { when } \quad \chi=1
\end{aligned}
$$

Coefficients $a, b$ and $c$ as well as $\varphi_{\max }$ can be calculated analytically for intermediate values of $\chi$ (appendix A). In Section 4, we shall show that $\chi$ is of the order of 0.1 or smaller in our experiments. In this case, numerics show that the optical contrast of the striations is given within an excellent approximation by formula (2) with $a=2$ and $b=c=1(\chi=0)$. That means that the contrast of the striations is maximum when $\varphi=\varphi_{\max }=\theta$, which gives a direct measurement of angle $\theta$. For example, the best fit of experimental data of Figure 2 to equation (2) for $\chi=0$ gives $\theta \approx 4^{\circ}$. This is the typical tilt angle of the columns in a zig or in a zag with respect to their average direction, in the pictures chosen for measuring the wavelength.

Finally, we measured wavelength $\Lambda$ of the zigzag in samples of different thicknesses. In Figure $3 \mathrm{a}$ we show the histogram of the wavelengths measured in a $100 \mu \mathrm{m}$ thick sample while in Figure $3 \mathrm{~b}$ we plot the average wavelength as a function of the thickness. In this figure, the size of the error bar is equal to the width of the Gaussian distribution that fits at best the histogram of the wavelengths. Finally, the solid line in Figure $3 \mathrm{~b}$ is the best fit to theoretical law (33) which will be established in Section $5(\Lambda$ proportional to $\sqrt{d})$.

\section{Elastic Energy and Linear Stability Analysis}

Let $u$ and $v$ be the column displacements along $x$ and $y$-axes (Fig. 4). The $x$-axis is perpendicular to the glass plates and the $z$-axis is parallel to the columns. The elastic energy density 


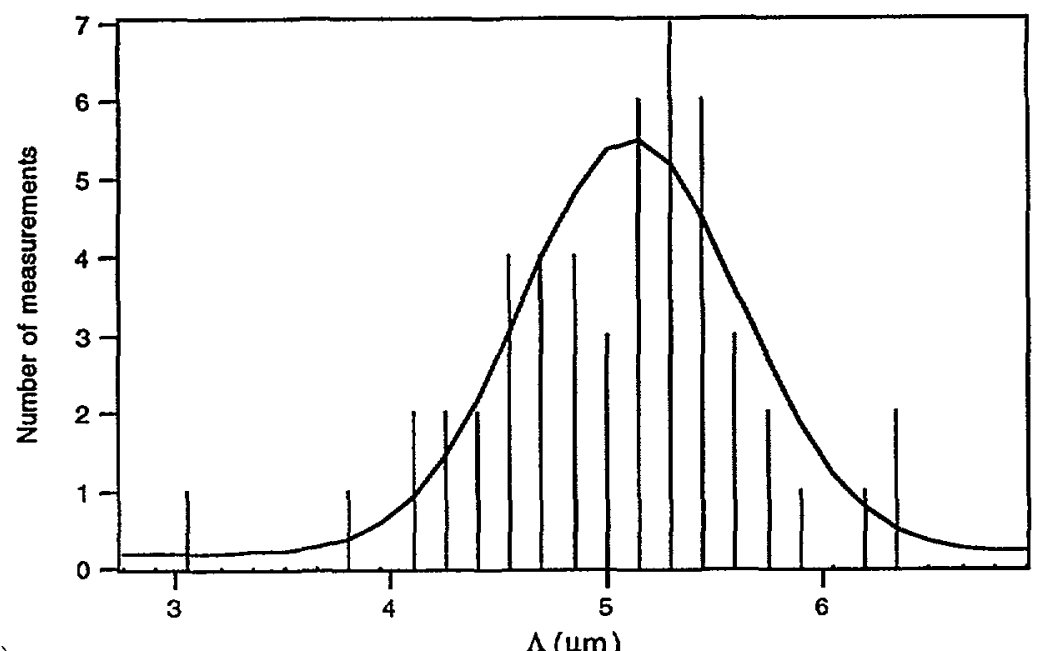

a)

$\Lambda(\mu \mathrm{m})$

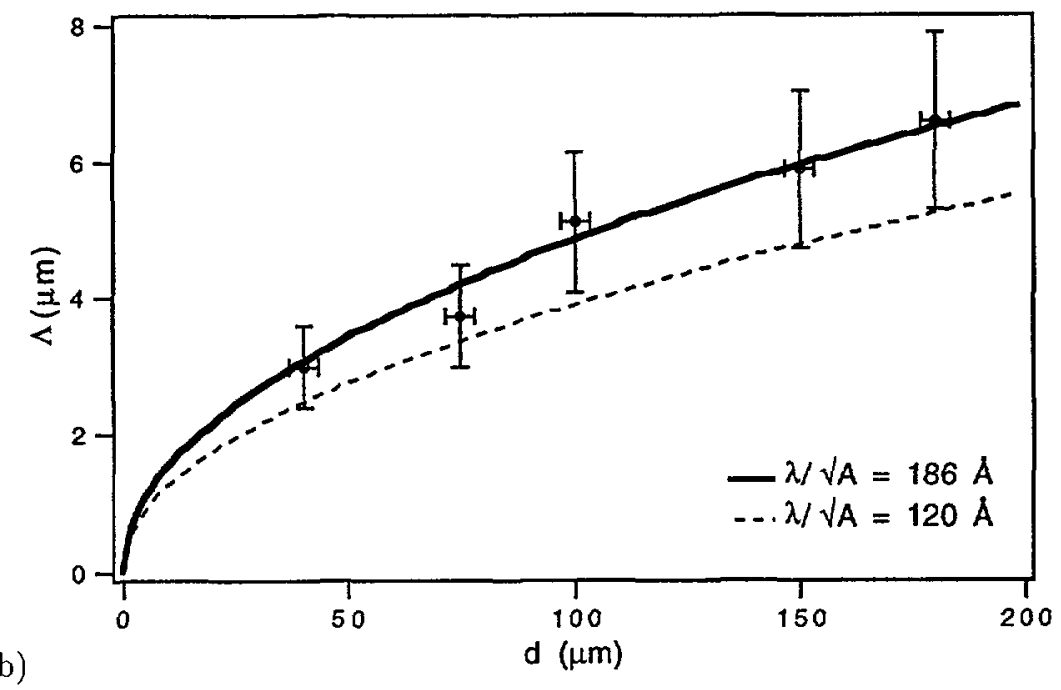

Fig. 3. - a) Histogram of the wavelengths measured in a $150-\mu$ m-thick sample. b) Wavelength $\Lambda$ of the striations as a function of thickness $d$. The solid line is the best fit to equation (33) with $\lambda / \sqrt{A}=186 \AA$.

can be written in the form [5]

$$
\rho f=\frac{1}{2} B_{1}\left(U_{x x}+U_{y y}\right)^{2}+\left(B_{2}-B_{1}\right)\left(U_{x x} U_{y y}-U_{x y}^{2}\right)+\frac{1}{2} K\left[\left(\frac{\partial^{2} u}{\partial z^{2}}\right)^{2}+\left(\frac{\partial^{2} v}{\partial z^{2}}\right)^{2}\right]
$$

In this expression, $B_{1}$ represents the compressibility modulus of the hexagonal array and $\left(B_{1}-B_{2}\right)$ is its shear modulus. In the following, we set $B_{1}=B,\left(B_{1}-B_{2}\right)=\frac{\mu}{2}$ and $A=\frac{1}{4} \frac{\mu}{B}$ for the elastic anisotropy. The curvature elastic constant of the columns is $K$ and the penetration length is $\lambda=\sqrt{K / B}$. If anharmonic terms are taken into account, distortions 


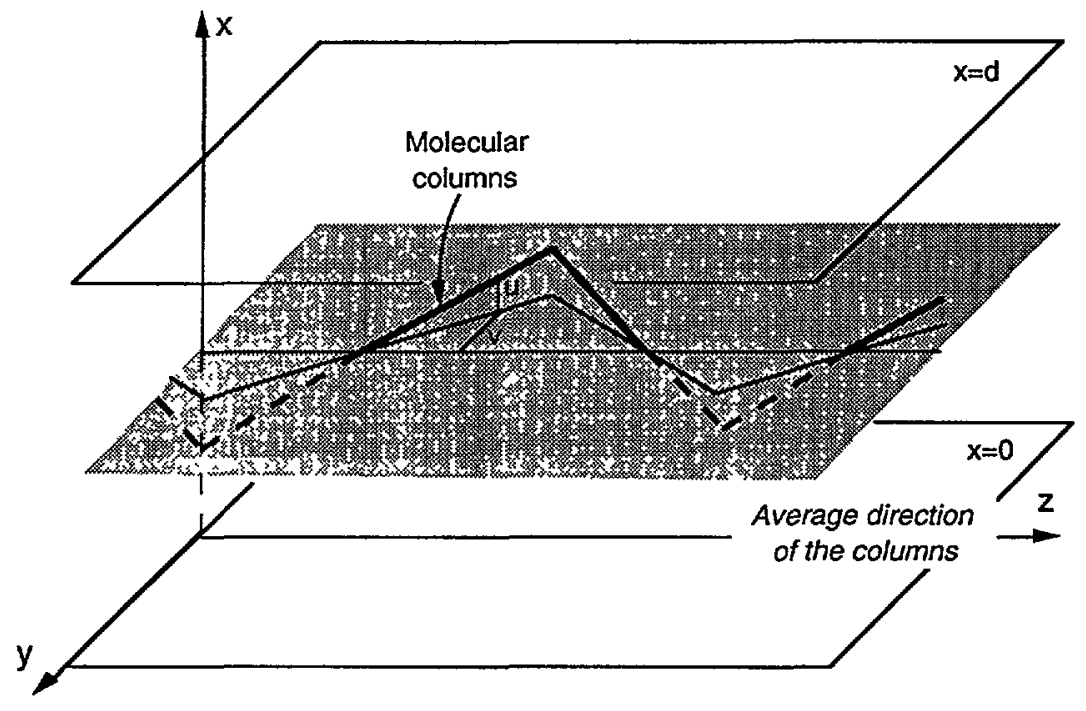

Fig. 4. - Definitions of displacements $u$ and $v$. The striations are parallel to $y$-axis.

$U_{\imath \jmath}$ are given by [5]:

$$
\begin{aligned}
U_{x x} & =\frac{\partial u}{\partial x}-\frac{1}{2}\left(\frac{\partial u}{\partial z}\right)^{2}+\frac{1}{2}\left(\frac{\partial v}{\partial x}\right)^{2} \\
U_{y y} & =\frac{\partial v}{\partial y}-\frac{1}{2}\left(\frac{\partial v}{\partial z}\right)^{2}+\frac{1}{2}\left(\frac{\partial u}{\partial y}\right)^{2} \\
U_{x y} & =\frac{1}{2}\left(\frac{\partial u}{\partial y}+\frac{\partial v}{\partial x}\right)-\frac{1}{2}\left(\frac{\partial u}{\partial x} \frac{\partial v}{\partial x}+\frac{\partial u}{\partial y} \frac{\partial v}{\partial y}+\frac{\partial u}{\partial z} \frac{\partial v}{\partial z}\right)
\end{aligned}
$$

In order to find the threshold of undulation of the columns, we use the same procedure as in reference [5]. Let $\gamma$ be the two-dimensional dilation of the hexagonal array. This dilation results from the density jump at the transition and from the decrease of the lattice parameter when the temperature decreases [13]. Since the sample thickness is maintained constant by two spacers, these two effects produce a net dilation of the hexagonal array. This dilation can relax, either by climb of transverse edge dislocation, or by forming column undulations in order to locally decrease the column spacing. In lyotropic systems, the plastic relaxation is ineffective, certainly because permeation is very difficult. By contrast, undulations or zigzags form very easily. To calculate the critical dilation $\gamma_{c}$ above which they occur, we must specify boundary conditions on the limiting surfaces. In the following, we shall assume that the columns do not undulate when they touch the glass plates. Assuming further that they strongly anchor on glass, we obtain

$$
u=\gamma x \text { and } \quad v=\gamma y \quad \text { at } \quad x=0 \text { and } x=d
$$

where $d$ is the sample thickness. 
In a linear analysis, the displacement field can be taken in the form (harmonic approximation):

$$
\begin{aligned}
& u=\gamma x+X(z) \sin \left(q_{x} x\right) \\
& v=\gamma y+Y(z) \sin \left(q_{x} x\right)
\end{aligned}
$$

where $q_{x}=\frac{\pi}{d}$ in order to satisfy boundary conditions (8). A straightforward calculation of the average energy defined to be $\langle\rho f\rangle_{d}=\frac{1}{d} \int_{0}^{d} \rho f \mathrm{~d} x$, gives:

$$
\begin{aligned}
\frac{4}{B}<\rho f>_{d}=8(1-A) \gamma^{2}+\lambda^{2}\left[\left(\frac{\partial^{2} X}{\partial z^{2}}\right)^{2}+\left(\frac{\partial^{2} Y}{\partial z^{2}}\right)^{2}\right]-2 \gamma(1-A)\left[\left(\frac{\partial X}{\partial z}\right)^{2}+\left(\frac{\partial Y}{\partial z}\right)^{2}\right] \\
+q_{x}^{2}\left[X^{2}+\gamma(1-A) Y^{2}\right]-\frac{q_{x}^{2}}{8} Y^{2}\left[\left(\frac{\partial X}{\partial z}\right)^{2}-(1-2 A)\left(\frac{\partial Y}{\partial z}\right)^{2}\right] \\
+\frac{A}{4} q_{x}^{2} X Y\left(\frac{\partial X}{\partial z}\right)\left(\frac{\partial Y}{\partial z}\right)+\frac{3 A}{4} q_{x}^{4} X^{2} Y^{2}+\frac{3}{16} q_{x}^{4} Y^{4}+\frac{3}{16}\left[\left(\frac{\partial X}{\partial z}\right)^{2}+\left(\frac{\partial Y}{z}\right)^{2}\right]^{2}
\end{aligned}
$$

where $\lambda=\sqrt{\frac{K}{B}}$ is the penetration length. To find the limit of absolute instability, we set:

$$
X(z)=u_{0} \sin \left(q_{z} z\right) \text { and } Y(z)=v_{0} \sin \left(q_{z} z\right)
$$

where $\Lambda=2 \pi / q_{z}$ is the undulation wavelength, and we calculate the minimal value of $\gamma$ for which the coefficients of the quadratic terms in $u_{0}^{2}$ and $v_{0}^{2}$ in free energy (10) vanish. These two terms read:

$$
\begin{aligned}
\lambda^{2} q_{z}^{4}+q_{x}^{2}-2 \gamma(1-A) q_{z}^{2} & =0 & & \text { (in } \left.u_{0}^{2}\right) \\
\lambda^{2} q_{z}^{4}+q_{x}^{2}\left[2 \gamma(1-A)+A(1-\gamma)^{2}\right]-2 \gamma(1-A) q_{z}^{2} & =0 & & \text { (in } \left.v_{0}^{2}\right)
\end{aligned}
$$

The first equation gives the threshold of undulation in the vertical $(x, z)$ plane:

$$
\Lambda_{u c}=2 \sqrt{\pi \lambda d} \quad \text { and } \quad \gamma_{u c}=\frac{\lambda q_{x}}{1-A}
$$

while the second gives the threshold of undulation in the horizontal $(y, z)$ plane:

$$
\Lambda_{v \mathrm{c}}=\frac{2 \sqrt{\pi \lambda d}}{\sqrt[4]{A}} \quad \text { and } \quad \gamma_{v \mathrm{c}}=\frac{\lambda q_{x}}{1-A} \sqrt{A}
$$

Because $A<1$, then $\gamma_{v c}<\gamma_{u c}$ and $\Lambda_{v c}>\Lambda_{u c}$. It means that undulations first appear in the horizontal plane with a slightly larger wavelength than in the vertical plane. In the following we set $\gamma_{v c}=\gamma_{c}$ and $\Lambda_{v c}=\Lambda_{c}$.

In the system chosen $\left(\mathrm{C}_{12} \mathrm{EO}_{6}+\right.$ water $), \lambda \approx 10 \AA[10,11] 0.025<A<0.075$ [16]. By taking $A \approx 0.05$, we calculate $\gamma_{\mathrm{c}} \approx 10^{-5}$ and $\Lambda_{\mathrm{c}} \approx 2 \mu \mathrm{m}$ for $d=75 \mu \mathrm{m}$. This very small value of the critical dilation explains why undulations spontaneously form in the samples when they are cooled down. Indeed, we know that the dilation coefficient of the hexagonal phase is of the order of a few $10^{-4} \mathrm{~K}^{-1}[13]$, so that we expect that the thermal dilation of the hexagonal array is of the order of $10^{-3}$ in our experiment. Thus, our measurements are always done very far from the threshold of instability so that using formula (13a) is questionnable. 


\section{Non Linear Regime: from Undulation to Zigzag}

As long as $\gamma<\gamma_{u c}$, one can assume that the columns only undulate in the horizontal plane. We shall see in Section 5 that this assumption remains reasonable even when $\gamma>\gamma_{u c}$. Consequently, we shall take the displacement in the following form:

$$
\begin{aligned}
& u=\gamma x \\
& v=\gamma y+\Psi(x) Y(z)
\end{aligned}
$$

In the harmonic approximation $\Psi(x)=\sin \left(q_{x} x\right)$ and $Y(z)=v_{0} \sin \left(q_{z} z\right)$. With this choice, we see that $\Psi$ is a dimensionless function of order unity, whereas $Y(z)$ has the dimension of a length (amplitude of the modulation). Let us emphasize that equation (14b) explicitly assumes that the columns keep a similar shape in the thickness of the sample.

The local density of free energy reads:

$$
\frac{2 \rho f}{B}=4(1-A) \gamma^{2}+\bar{A} \Psi_{, x}^{2} Y^{2}-2(1-A) \gamma \Psi^{2} Y_{, z}^{2}+\frac{1}{4} \Psi^{4} Y_{, z}^{4}+\lambda^{2} \Psi^{2} Y_{, z z}^{2}
$$

where $\bar{A}=2(1-A) \gamma+A(1-\gamma)^{2} \approx A$ when $\gamma \ll 1$ and ", $x$ " denotes the first derivative with respect to $x$, etc. In this expression, terms like $\Psi_{, x}^{4} Y^{4}$ and $\Psi^{2} \Psi_{, x}^{2} Y^{2} Y_{, z}^{2}$ have been neglected, an approximation which can be fully justified a posteriori. The minimization of $f$ with respect to $Y$ and $\Psi$ gives the two following differential equations:

$$
\begin{aligned}
2 \lambda^{2} \Psi^{2} Y_{, z z z z}+4 \gamma(1-A) \Psi^{2} Y_{, z z}+2 \bar{A} \Psi_{, x}^{2} Y-\Psi^{4}\left(Y_{, z}^{3}\right)_{, z} & =0 \\
2 \bar{A} Y^{2} \Psi_{x x}+\left[4 \gamma(1-A) Y_{z}^{2}-2 \lambda^{2} Y_{, z z}^{2}\right] \Psi-Y_{z}^{4} \Psi^{3} & =0
\end{aligned}
$$

In order to find an approximate solution to equations $(16 \mathrm{a}, \mathrm{b})$ we use a mathematical method developped by Singer in smectics [17]. The procedure is as follows (see appendix C for detailed calculations): for a given value of $\Lambda$, we search for the solution $Y(z)$ of equation (16a) in the midplane $x=d / 2$ in which $\Psi_{, x}=0$ and we keep this form in the whole domain. Then, we determine $\Psi(x)$ by integrating equation (16b) in which we replace $Y(z)$ and its derivatives by their mean values over the wavelength. Finally, we calculate the mean elastic energy $\left.\langle E\rangle=\ll \rho f>_{d}\right\rangle_{\Lambda}$ and we minimize it with respect to the wavelength $\Lambda$.

In general, this problem must be solved numerically. Functions $Y(z), \Psi(x)$ are plotted in Figure 5 far from the threshold of instability. While $Y(z)$ and $\Psi(x)$ vary like a sinus for $\gamma / \gamma_{c}$ close to 1 , they respectively approach a triangle and a trapezium when $\gamma / \gamma_{v c}$ is large. In this limit, the columns form a zigzag of constant amplitude in the thickness of the sample, except in two twist boundary layers close to the glass plates, in which the amplitude of the undulation vanishes. In the following we call $\chi$ their relative thickness with respect to the half sample thickness:

$$
\chi=\frac{\pi}{2} \frac{\Psi(d / 2)}{\Psi_{, x}(0)}
$$

and $\theta$ the maximal tilt angle of the columns with respect to the $z$-axis in the middle of the sample:

$$
\theta=\left(\frac{\partial v}{\partial z}\right)_{\max }=\Psi(d / 2) Y_{z}(\Lambda / 4)
$$

The evolution of this two quantities is shown in Figure 6 as a function of $\gamma$. We also plotted $\Lambda$ and the maximum curvature $\kappa$ of the columns:

$$
\kappa=\left(\frac{\partial^{2} v}{\partial z^{2}}\right)_{\max }=\Psi(d / 2) Y_{, z z}(0)
$$




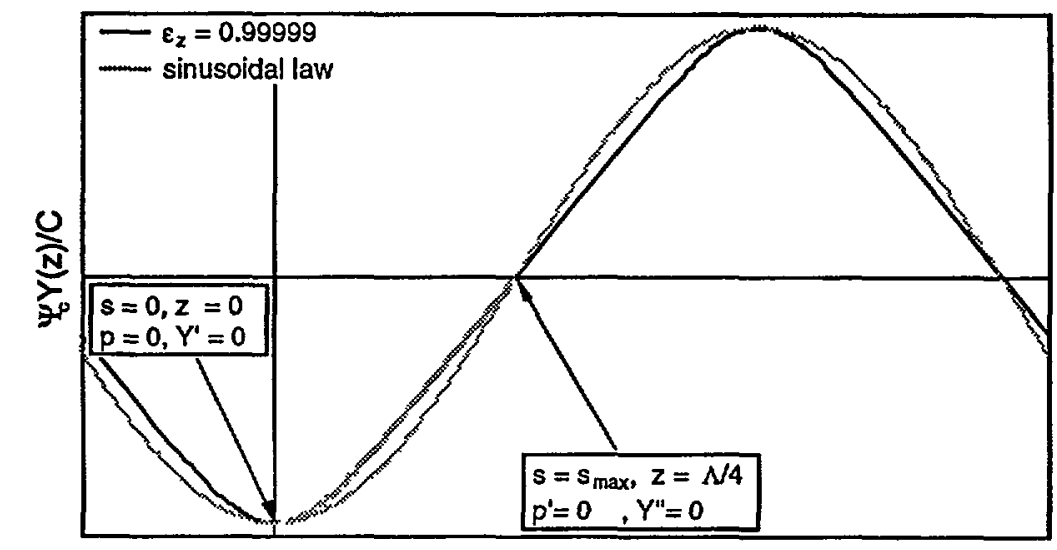

a) $z / \Lambda$

b)

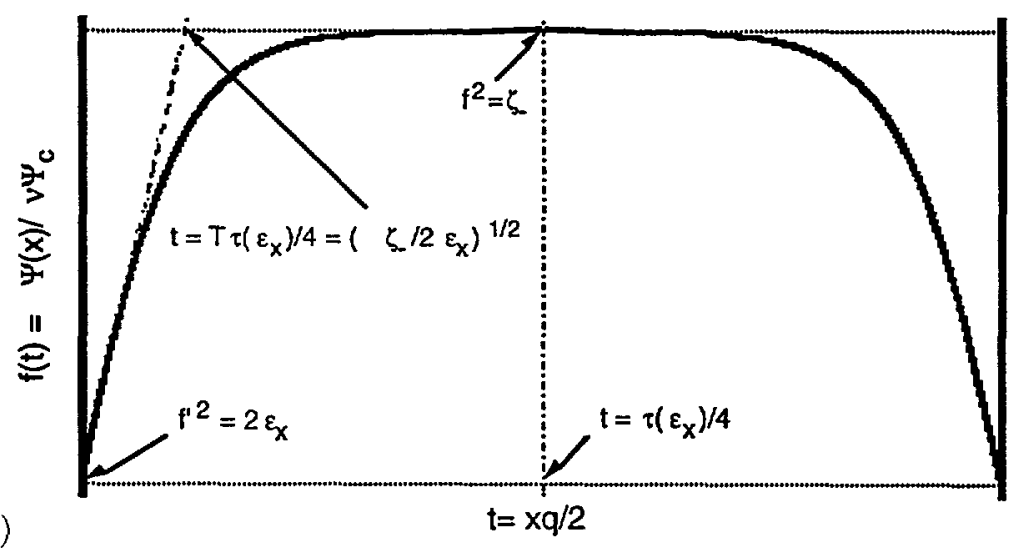

Fig. 5. - The two functions a) $Y(z)$ and b) $\Psi(x)$ give respectively the shape of the columns and the amplitude of their deformation in the sample thickness (see appendix $\mathrm{C}$ for the notations and the detailed calculations).

This figure clearly shows that these quantities quickly reach their asymptotic behaviors when $\gamma$ increases. These behaviors can be found analytically (appendix $\mathrm{C}$ ) and read:

$$
\begin{aligned}
\Lambda & =\sqrt{\frac{2 \sqrt{3}}{\pi}} \Lambda_{\mathrm{c}} \approx 1.05 \Lambda_{\mathrm{c}} \\
\theta & =2 \sqrt{\gamma(1-A)} \approx 2 \sqrt{\gamma} \\
\chi & =\sqrt{\frac{\pi}{2 \sqrt{3}} \sqrt{\frac{\gamma_{\mathrm{c}}}{\gamma}}} \\
\kappa & =\frac{2 \gamma(1-A)}{\lambda}
\end{aligned}
$$

These formula are important for many reasons. First, equation (20) gives a direct estimation of dilation $\gamma$ as long as $\theta$ is known, independently of the thickness and of the other physical constants. For instance, $\gamma \approx 1.2 \times 10^{-3}$ for the value of $\theta$ found experimentally $\left(\theta=4^{\circ}\right.$, see Sect. 1). This result shows that $\gamma \gg \gamma_{c}$ experimentally so that previous formulas are 

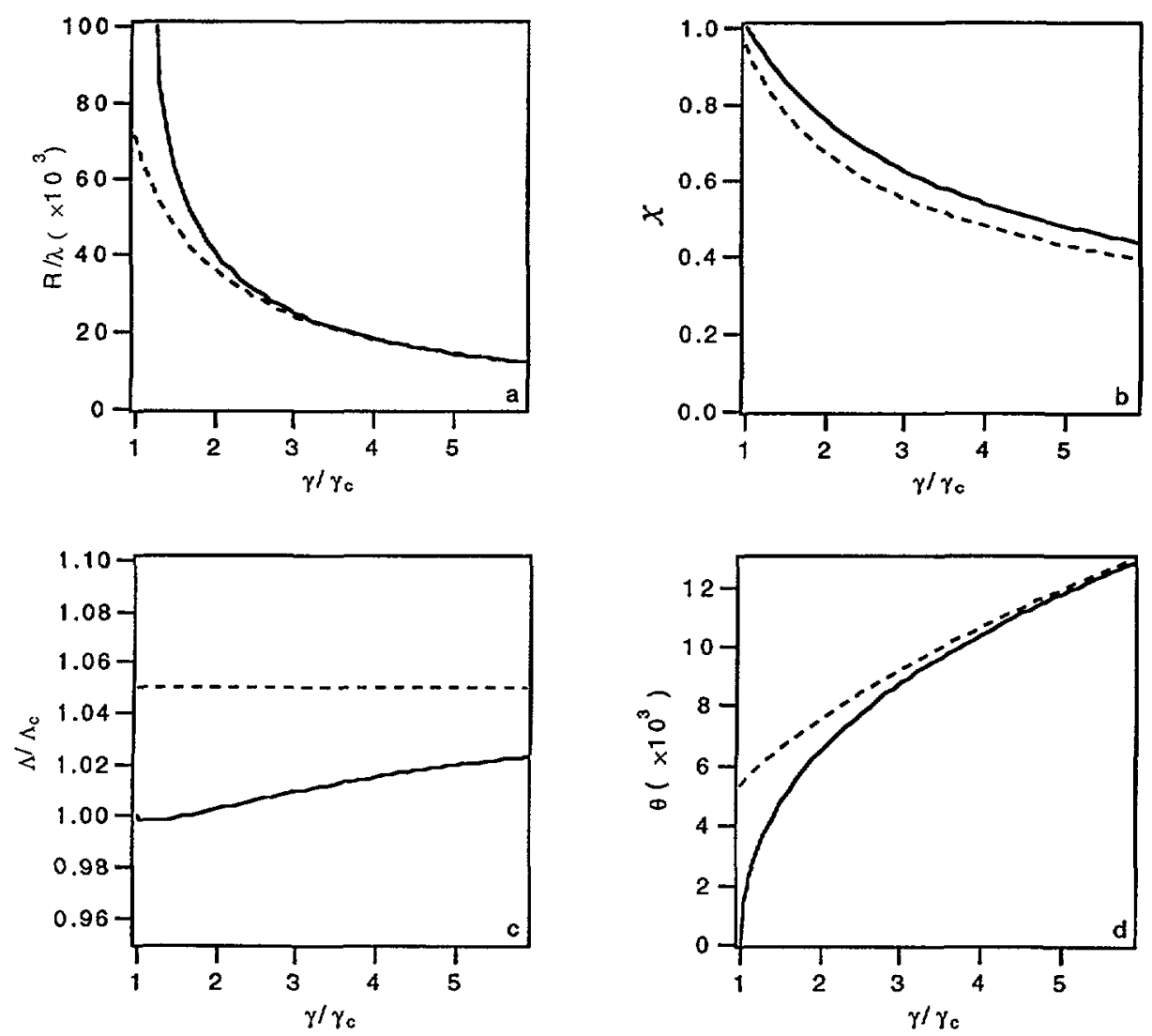

Fig. 6. - Wavelength $\Lambda$, tilt angle $\theta$, relative thickness $\chi$ and minimum radius of curvature $R=\kappa^{-1}$ as a function of dilation $\gamma$ calculated with $d=100 \mu \mathrm{m}, \lambda=10 \AA$ and $A=0.05$.

usable. Note that equation (20) can be found again very easily by writing that the density of energy (6) is minimal when $U_{x x}+U_{y y}=2 \gamma-\frac{1}{2} \theta^{2}=0$ (on condition that the shear energy and the curvature energy are negligible, i.e. $A=K=0$ ). The same argument was used by Singer [17] and Sornette [18] to calculate the angle of the zigzag in smectics: in this case, the result $(\theta=\sqrt{2 \gamma})$ is different because the dilation is uniaxial. Finally, let us emphasize that the wavelength does not depend on $\gamma$ and remains equal to $\Lambda_{c}$ within $5 \%$.

In the following section, we show that these results can be found again from a simplified model. We only assume that the columns form a zigzag in the sample thickness, a result obtained from the Singer model presented in this section. On the other hand, we shall no longer assume that the columns remain in the horizontal plane.

\section{Zigzag Regime: a Simplified Model}

In the preceding section, we assumed that $u=0$ to simplify. We found that the in-plane undulation rapidly tends to a zigzag above the critical threshold.

In this section, we assume that $\gamma / \gamma_{\mathrm{c}}$ is large and we no longer assume that $u=0$. In this case, previous calculations become cumbersome and a simplified model is preferable. 
We first assume that columns form a simple zigzag as shown in the preceding section. In this limit, columns are rectilinear in a zig and in a zag with a wall of curvature in between. If the misfit angle of the columns between a zig and a zag is large enough (a few degrees), the width of the wall (of the order of $\lambda$ over the misfit angle) is much smaller than the wavelength of the zigzag. In this case, the free energy per unit surface area contains two terms, the wall energy and the energy of compression and shear of the hexagonal array in a zig or in a zag. Between two walls of curvature the displacement field can be written in the form:

$$
\begin{aligned}
& u=\gamma x+\Psi(x) \alpha z \\
& v=\gamma y+\Psi(x) \theta z
\end{aligned}
$$

where $\Psi(x)$ is a function which must vanish at $x=0$ and $x=d$. According to previous calculations, we shall take:

$$
\Psi(x)= \begin{cases}\sin \left(\frac{\pi}{\chi} \frac{x}{d}\right) & 0<x<d \frac{\chi}{2} \\ 1 & d \frac{\chi}{2}<x<d\left(1-\frac{\chi}{2}\right) \\ \sin \left[\frac{\pi}{\chi}\left(1-\frac{x}{d}\right)\right] & d\left(1-\frac{\chi}{2}\right)<x<d\end{cases}
$$

with $0<\chi<1$. The two angles $\alpha$ and $\theta$ are found by minimizing free energy (6) to which the wall energy is added. With our definition of angles $\alpha$ and $\theta$, the misfit angle between columns is equal to $2 \sqrt{\alpha^{2}+\theta^{2}} \Psi(x)$, so that the energy of a wall per unit length along $y$ equals [19]:

$$
\begin{aligned}
E_{\mathrm{wall}} & =\frac{2}{3} B \lambda\left(\alpha^{2}+\theta^{2}\right)^{3 / 2} \int_{0}^{d}[\Psi(x)]^{3} \mathrm{~d} x \\
& =\frac{8}{9 \pi} B \lambda d\left[\chi+\frac{3 \pi}{4}(1-\chi)\right]\left(\alpha^{2}+\theta^{2}\right)^{3 / 2}
\end{aligned}
$$

We can now calculate from equations $(6,24,25)$ the average energy per unit surface area of the sample:

$$
\begin{aligned}
\frac{4}{B d}<E>= & \text { Cst }-2 \gamma(2-\chi)(1-A)\left(\alpha^{2}+\theta^{2}\right)+\frac{1}{12}\left(\alpha^{2}+A \theta^{2}\right) \frac{q_{x}^{2} L^{2}}{\chi} \\
& +\frac{1}{2}\left[1-\frac{5}{8} \chi\right]\left(\alpha^{2}+\theta^{2}\right)^{2}+\frac{32}{9 \pi} \frac{\lambda}{L}\left[\chi+\frac{3 \pi}{4}(1-\chi)\right]\left(\alpha^{2}+\theta^{2}\right)^{3 / 2}
\end{aligned}
$$

where $L$ is the length of a $\mathrm{zag}(\Lambda=2 L)$ and $q_{x}=\pi / d$. Minimization with respect to $L$ gives:

$$
L=\left[\frac{16 \lambda d^{2}}{\pi^{2}}\right]^{1 / 3} \chi^{1 / 3}\left[1-\chi\left(1-\frac{4}{3 \pi}\right)\right]^{1 / 3} \frac{\left(\alpha^{2}+\theta^{2}\right)^{1 / 2}}{\left(\alpha^{2}+A \theta^{2}\right)^{1 / 3}}
$$

and

$$
\begin{aligned}
\frac{4}{B d}<E>= & \text { Cst }-2 \gamma(2-\chi)(1-A)\left(\alpha^{2}+\theta^{2}\right)+\frac{1}{2}\left[1-\frac{5}{8} \chi\right]\left(\alpha^{2}+\theta^{2}\right)^{2} \\
& +(2 \pi)^{2 / 3}\left(\frac{1}{\chi}\right)^{1 / 3}\left(\frac{\lambda}{d}\right)^{2 / 3}\left[1-\chi\left(1-\frac{4}{3 \pi}\right)\right]^{2 / 3}\left(\alpha^{2}+A \theta^{2}\right)^{1 / 3}\left(\alpha^{2}+\theta^{2}\right)
\end{aligned}
$$


Minimization of this function with respect to $\chi, \theta$ and $\alpha$ can be done numerically using Mathematica. This calculation shows that $\alpha=0$ at the minimum which means that the columns remain parallel to the glass plates.

In order to compare this model with the previous one, we calculated $\theta, \chi$ and $\Lambda$ as a function of $\gamma$ for $d=100 \mu \mathrm{m}$ by taking $\lambda=10 \AA$ and $A=0.05$. We found that for $\gamma>10^{-3}$,

$$
\begin{aligned}
\Lambda & \approx(1.020 \pm 0.007) \Lambda_{\mathrm{c}} \\
\theta & \approx(1.92 \pm 0.01) \sqrt{\gamma} \\
\chi & \approx(1.071 \pm 0.006) \sqrt{\frac{\gamma_{\mathrm{c}}}{\gamma}} \\
\kappa & =(1.84 \pm 0.02) \frac{\gamma}{\lambda}
\end{aligned}
$$

These results are in good agreement with the previous model within $5 \%$. Similar computations have been done for other values of the thickness and confirm that the wavelength $\Lambda$ is given within a very good approximation $(5 \%)$ by its expression at the threshold, namely

$$
\Lambda \approx \frac{2 \sqrt{\pi \lambda d}}{\sqrt[4]{A}}
$$

in agreement with equation (19).

\section{Discussion}

We can now estimate the ratio $\lambda / \sqrt{A}$ from the best fit of experimental data of Figure $3 \mathrm{~b}$ to equation (33). We obtain:

$$
\frac{\lambda}{\sqrt{A}}=(186 \pm 60) \AA
$$

By taking the extreme values of $A$ given by X-ray experiments of Clerc [16] $(0.025<A<0.075)$ we get:

$$
\lambda=(40 \pm 20) \AA
$$

This value is of the same order of magnitude as that given by X-ray diffraction $(\lambda \approx 20 \AA)[16]$. On the other hand, it is larger than the value $(\lambda \approx 6 \AA)$ which we found previously from the measurements of the energy of the dislocations. Several factors could explain this discrepancy: first, wavelength measurements have been done a few degrees below the transition temperature whereas previous experiment was performed at the transition temperature. Second, the previous estimation was obtained via a dislocation model which we know to be imperfect, insofar as there is no precise calculation of the core size and of the corresponding core energy. Finally, we are not completely sure that the columns do not glide on the glass plates or that no screw dislocation nucleates: such phenomena would increase the wavelength so that $\lambda$ should be smaller than the value given in this article.

Consequently, the single, but important conclusion, that can be stressed from all these measurements is that $\lambda$ is comparable to the columns spacing. We also emphasize that the present measurement is nonlocal, in contrast to previous ones (dislocations and X-rays) that were performed at a submicrometric scale. That means that columns are not correlated at large distance in the static limit.

One could ask whether the same conclusions can be drawn in thermotropic columnar mesophases. So far, no definite answer can be given because thermal striations do not appear so 


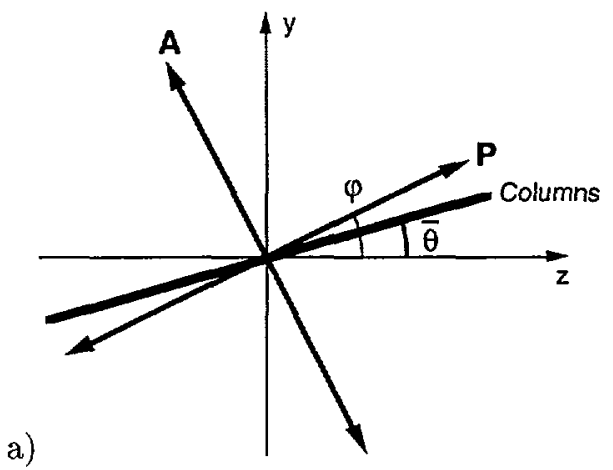

b)

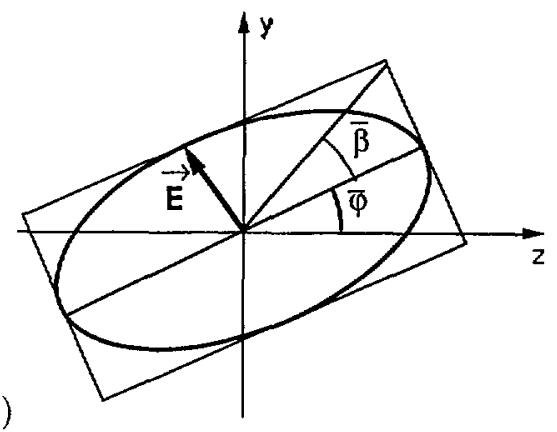

Fig. 7. - a) Disposition of the polarizer and of the analyzer with respect to the columns and to the coordinate axes; b) Definition of the two angles $\bar{\beta}$ and $\bar{\varphi}$ characterizing an elliptically polarized planar wave.

easily in thermotropic systems as in lyotropics. In fact, we did not observe striations in planar domains grown in directional solidification. A possible explanation would be that stresses generated by a very slow dilation of the hexagonal array relax in thermotropic liquid crystals by permeation or climb of transverse edge dislocations. Consequently, thermal striations are more difficult to produce in these systems than in lyotropic liquid crystals in which plastic stress relaxation is very slow (several months are necessary for the striations to disappear: this is perhaps due, in part, to the fact that the samples dry a little in the same time). A possible experiment in discotic liquid crystals would be to dilate planar samples at different velocities, in order to test the role of frequency and of plastic processes in the determination of the elastic constants. By contrast, similar experiments are likely to be very difficult to do in lyotropic systems because of the difficulty to prepare planar monodomains without pre-existent undulations.

\section{Acknowledgments}

This work was supported by C.N.E.S. contract N 94/0253. We thank P. Ribière for fruitful comments concerning optics.

\section{Appendix A}

\section{Optical Contrast of the Stripes Between Crossed Polarizers}

In order to calculate the optical contrast of the stripes between crossed polarizers, we assume that the columns are parallel to the glass plates and form a zigzag. Let $\bar{\theta}(x)$ be the tilt angle of the columns with respect to the $z$-axis and $\varphi$ the angle between the polarizer and the $z$-axis (Fig. 7a). For simplicity, we assume that

$$
\bar{\theta}(x)= \begin{cases}\theta \sin \left(\frac{\pi}{\chi} \frac{x}{d}\right) & 0<x<d \frac{\chi}{2} \\ \theta & d \frac{\chi}{2}<x<d\left(1-\frac{\chi}{2}\right) \\ \theta \sin \left[\frac{\pi}{\chi}\left(1-\frac{x}{d}\right)\right] & d\left(1-\frac{\chi}{2}\right)<x<d\end{cases}
$$


In this expression, $\chi$ is a dimensionless factor ranging between 0 and 1 and $d$ is the sample thickness. At $x=0$, the light is linearly polarized. On the other hand, it becomes elliptically polarized in the sample because the optical axis rotates along the $x$-axis. In this case, the end of the electric field $\mathbf{E}$ traces out an elliptical path in time which can be described by two angles $\bar{\varphi}$ and $\bar{\beta}$ (Fig. 7b) such as

$$
\begin{aligned}
& E_{z}(x)=\cos (\bar{\beta}) \cos (\bar{\varphi}) \cos (\omega t)-\sin (\bar{\beta}) \sin (\bar{\varphi}) \sin (\omega t) \\
& E_{y}(x)=\cos (\bar{\beta}) \sin (\bar{\varphi}) \cos (\omega t)-\sin (\bar{\beta}) \cos (\bar{\varphi}) \sin (\omega t)
\end{aligned}
$$

It can be shown [20] that $\alpha$ and $\beta$ are solutions of the two following differential equations:

$$
\begin{array}{r}
\frac{\mathrm{d}(2 \bar{\varphi})}{\mathrm{d} x}=\frac{\Delta \Phi}{d} \tan (2 \bar{\beta}) \cos (2 \bar{\varphi}-2 \bar{\theta}) \\
\frac{\mathrm{d}(2 \bar{\beta})}{\mathrm{d} x}=-\frac{\Delta \Phi}{d} \sin (2 \bar{\varphi}-2 \bar{\theta})
\end{array}
$$

with boundary conditions

$$
\bar{\beta}=0 \text { and } \bar{\varphi}=\varphi \quad \text { at } \quad x=0
$$

Close to the onset of instability, $\chi=1$ and angle $\bar{\theta}$ is very small. In this limit, the equations $(A .3 a, b)$ can be solved analytically by assuming that $\bar{\varphi}$ is small. We find:

$$
\begin{aligned}
& \bar{\varphi}+\bar{\beta}=\mathcal{A} \cos \left(\frac{\Delta \Phi}{d}+\Phi\right)+\mathcal{B} \cos \left(q_{x} x\right)+\mathcal{C} \sin \left(q_{x} x\right) \\
& \bar{\varphi}-\bar{\beta}=\mathcal{A} \sin \left(\frac{\Delta \Phi}{d}+\Phi\right)+\mathcal{D} \cos \left(q_{x} x\right)+\mathcal{E} \sin \left(q_{x} x\right)
\end{aligned}
$$

and

$$
\begin{aligned}
\mathcal{E} & =\mathcal{C}=-\frac{(\Delta \Phi)^{2} \theta}{\pi^{2}-(\Delta \Phi)^{2}}, \quad \mathcal{D}=-\mathcal{B}=\frac{\pi(\Delta \Phi) \theta}{\pi^{2}-(\Delta \Phi)^{2}} \\
\mathcal{A} \cos \Phi & =\varphi+\mathcal{D}, \quad \mathcal{A} \sin \Phi=\varphi-\mathcal{D}
\end{aligned}
$$

with $q_{x}=\pi / d$ and $\Delta \Phi=2 \pi d \Delta n / \bar{\lambda}$. From these formulas, we calculate:

$$
\begin{aligned}
& \bar{\varphi}_{\text {out }}=\bar{\varphi}(x=d)=\mathcal{D} \sin (\Delta \Phi)+\varphi \cos (\Delta \Phi) \\
& \bar{\beta}_{\text {out }}=\bar{\beta}(x=d)=\mathcal{D}[\cos (\Delta \Phi)+1]-\varphi \sin (\Delta \Phi)
\end{aligned}
$$

which allows us to find $\mathbf{E}_{\text {out }}$ from equations (A.2a,b). With the electric field projection onto the analyzer being given by:

$$
E_{\mathrm{out}}^{\mathrm{A}}=E_{z}^{\mathrm{out}} \sin (\varphi)-E_{y}^{\mathrm{out}} \cos (\varphi)
$$

we thus obtain the light intensity passing through the crossed polarizers:

$$
I_{\text {out }}^{\mathrm{A}}=\left\langle E_{\text {out }}^{\mathrm{A}}\right\rangle^{2}=I_{0}\left[\varphi \sin \left(\frac{\Delta \Phi}{2}\right)-\frac{\pi(\Delta \Phi) \theta}{\pi^{2}-(\Delta \Phi)^{2}} \cos \left(\frac{\Delta \Phi}{2}\right)\right]^{2}
$$

and the zigzag contrast between crossed polarizers:

$$
\begin{aligned}
\mathcal{C}_{\perp}(\chi=1) & =\frac{I_{\text {out }}^{\mathrm{A}}(\theta)-I_{\text {out }}^{\mathrm{A}}(-\theta)}{I_{\text {out }}^{\mathrm{A}}(\theta)+I_{\text {out }}^{\mathrm{A}}(-\theta)} \\
& =\frac{\pi \Delta \Phi \sin (\Delta \Phi)\left[\pi^{2}-(\Delta \Phi)^{2}\right] \theta \varphi}{\left[\pi^{2}-(\Delta \Phi)^{2}\right]^{2} \sin ^{2}\left(\frac{\Delta \Phi}{2}\right) \varphi^{2}+\pi^{2}(\Delta \Phi)^{2} \cos ^{2}\left(\frac{\Delta \Phi}{2}\right) \theta^{2}}
\end{aligned}
$$




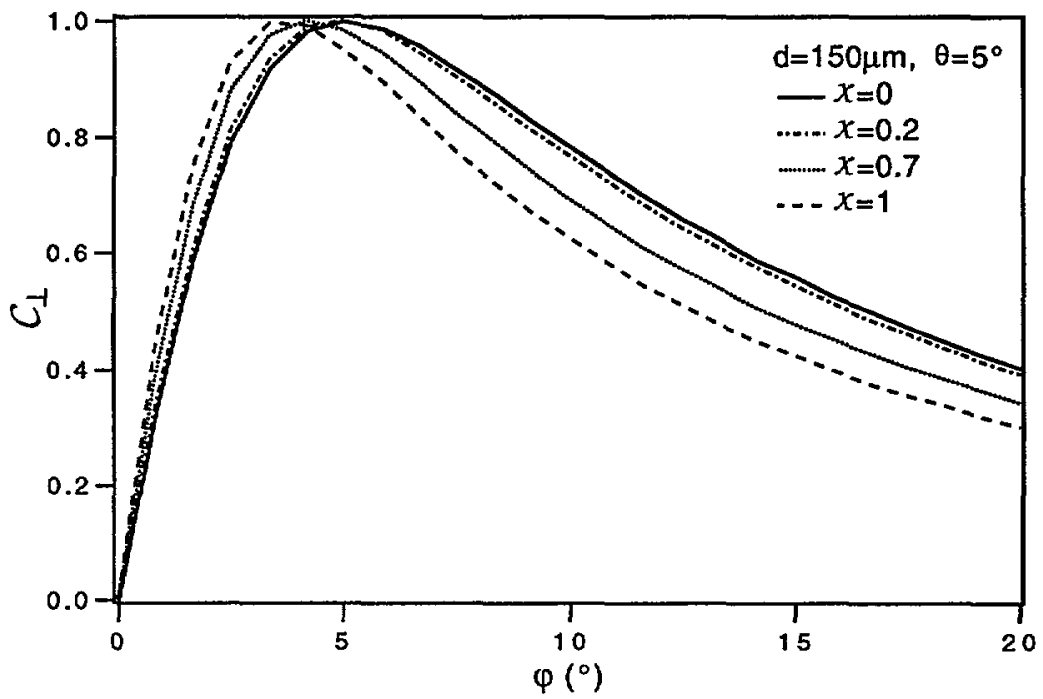

Fig. 8. - Optical contrast $\mathcal{C}_{\perp}$ as a function of angle $\varphi$ between the polarizer and the average direction of the columns for different values of parameter $\chi\left(d=150 \mu \mathrm{m}\right.$ and $\left.\theta=5^{\circ}\right)$.

The optical contrast can also be exactly calculated when $\chi=0$. It reads:

$$
\mathcal{C}_{\perp}(\chi=1)=\frac{\sin ^{2}[2(\theta-\varphi)]+\sin ^{2}[2(\theta+\varphi)]}{\sin ^{2}[2(\theta-\varphi)]-\sin ^{2}[2(\theta+\varphi)]} \approx \frac{2 \theta \varphi}{\varphi^{2}+\theta^{2}}
$$

By contrast, numerics are necessary for solving equation (A.3a,b) for intermediate values of $\chi$ and large values of angle $\varphi$. For example, we give in Figure 8 different curves $\mathrm{C}_{\perp}(\chi, \varphi)$ obtained by taking $\theta=5^{\circ}$ and $d=150 \mu \mathrm{m}$. We see that $\mathcal{C}_{\perp}(\chi) \approx \mathcal{C}_{\perp}(\chi=0)$ as long as $\chi<0.2$.

\section{Appendix B}

\section{Birefringence Measurement}

To determine the birefringence of the hexagonal mesophase, we used a planar sample of thickness $d=150 \mu \mathrm{m}$. We did our measurements in regions of the sample where the amplitude of the striations was very small (typically $\theta<1^{\circ}$ ). As striations tend to very slowly disappear, the samples are stored, undisturbed during one month.

To determine the phase shift $\Delta \Phi$, we measured the transmitted intensity between crossed polarizers $I_{\perp}$ as a function of angle $\varphi$ between the polarizer and the optical axis. If the distortions are negligible $(\theta \approx 0)$, we have simply:

$$
I_{\perp}=I_{0}[\sin (2 \varphi)]^{2}\left(\sin \frac{\Delta \Phi}{2}\right)^{2}
$$

In this expression $I_{0}$ can be determined by measuring the transmitted intensity $I_{\|}$between parallel polarizers at $\varphi=0$ :

$$
I_{0}=I_{\mid l}(\varphi=0)
$$




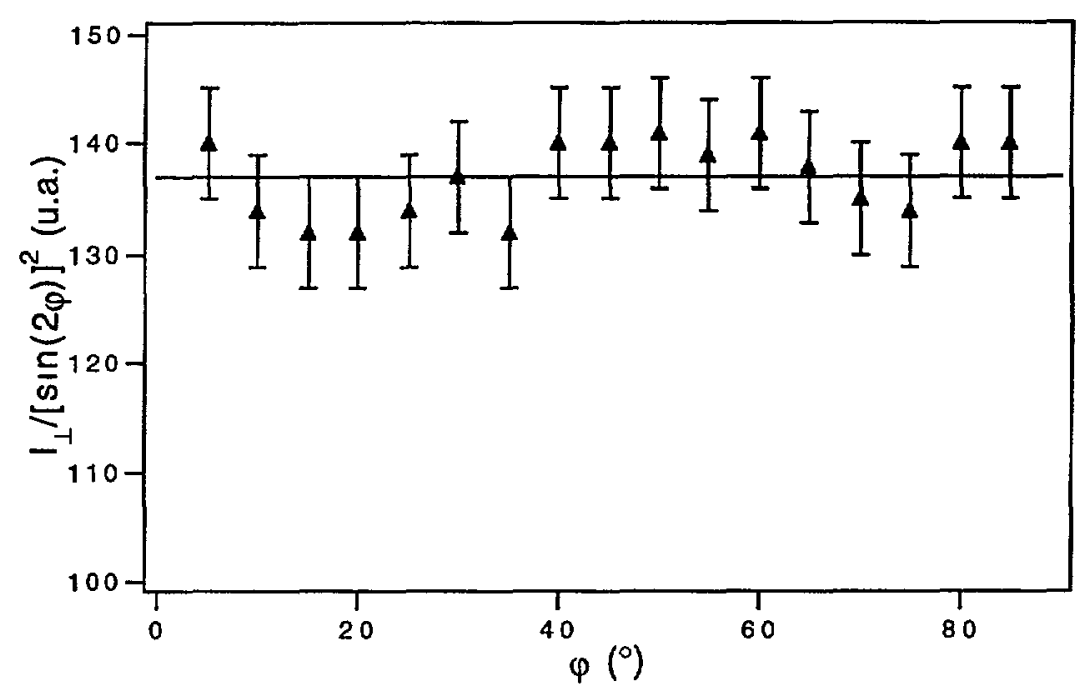

Fig. 9. - Transmitted intensity $I_{\perp} /[\sin (2 \varphi)]^{2}$ across a planar sample of thickness $d=150 \mu \mathrm{m}$ versus angle $\varphi$ in monochromatic light $(\bar{\lambda}=542 \mathrm{~nm})$.

In Figure 9, we plotted $I_{\perp} /[\sin (2 \varphi)]^{2}$ as a function of angle $\varphi$. Measurements were done in monochromatic light $(\bar{\lambda}=542 \pm 5 \mathrm{~nm})$ with a WV-BP500 Panasonic videocamera that we know to be linear in our range of light intensity. The condensor of the microscope was removed for obtaining parallel rays. Within experimental errors, this quantity is constant and equals $137 \pm 5$ in arbitrary units. We also measured $I_{0}$ which we found close to 149 in the same units. We thus obtained from equation (C.1) $\Delta \Phi \approx 2.56$, which gives $\Delta n \approx 1.5 \times 10^{-3}$ at $25^{\circ}$.

\section{Appendix C}

\section{Nonlinear Model}

In order to describe the undulations of the columns in the horizontal plane, we take the displacement in the following form:

$$
\begin{aligned}
& u=\gamma x \\
& v=\gamma y+\Psi(x) Y(z)
\end{aligned}
$$

These formulas assume that the columns do not undulate in the vertical plane and that the shape of the columns remains similar in the whole sample thickness.

We also assume that the columns do not undulate on the glass plates:

$$
\begin{aligned}
& \Psi(0)=0 \\
& \Psi(d)=0
\end{aligned}
$$

The distortions can be calculated from formulas $(5 \mathrm{a}-\mathrm{c})$ and read:

$$
U_{x x}^{2}=\gamma^{2}+\frac{1}{4} \Psi_{x}^{4} Y^{4}+\gamma \Psi_{x}^{2} Y^{2}
$$




$$
\begin{aligned}
& U_{y y}^{2}= \gamma^{2}+\frac{1}{4} \Psi^{4} Y_{, z}^{4}-\gamma \Psi^{2} Y_{z}^{2} \\
& U_{x x} U_{y y}= \gamma^{2}+\frac{\gamma}{2} \Psi_{x}^{2} Y^{2}-\frac{\gamma}{2} \Psi^{2} Y_{z}^{2}-\frac{\gamma}{4} \Psi_{x}^{2} \Psi^{2} Y^{2} Y_{z}^{2} \\
& U_{x y}^{2}=\frac{(1-\gamma)^{2}}{4} \Psi_{x}^{2} Y^{2}
\end{aligned}
$$

The substitution in equation (4) gives the local density of free energy (Eq. (13)):

$$
\tilde{f}=\frac{2 \rho f}{B}=4(1-A) \gamma^{2}+\bar{A} \Psi_{x}^{2} Y^{2}-2(1-A) \gamma \Psi^{2} Y_{, z}^{2}+\frac{1}{4} \Psi^{4} Y_{z}^{4}+\lambda^{2} \Psi^{2} Y_{z z}^{2}
$$

where $\bar{A}=2(1-A) \gamma+A(1-\gamma)^{2}$.

The minimization of the free energy with respect to $Y(z)$ and $\Psi(x)$ leads to relations (14a) and $(b)$ :

$$
\begin{aligned}
2 \lambda^{2} \Psi^{2} Y_{, z z z z}+4 \gamma(1 s-A) \Psi^{2} Y_{, z z}+2 \bar{A} \Psi_{, x}^{2} Y-\Psi^{4}\left(Y_{, z}^{3}\right)_{, z} & =0 \\
2 \bar{A} Y^{2} \Psi_{x x}+\left[4 \gamma(1-A) Y_{z}^{2}-2 \lambda^{2} Y_{z z}^{2}\right] \Psi-Y_{z}^{4} \Psi^{3} & =0
\end{aligned}
$$

The problem is now to find approximate solutions $Y(z)$ and $\Psi(x)$ to this set of differential equations. The following calculations constitute a generalization of the model of Singer [17] to columnar mesophases.

\section{Shape of the Undulation $Y(z)$ in the Plane of the Glass Plates}

We have already assumed that $Y$ depends only on $z$, so that we can, without other assumption, solve equation (C.6a) in the midplane $x=d / 2$ where $\Psi_{, x}=0$. To take into account the fact that $\Psi$ does not remain constant in the whole sample thickness, we give to $\Psi$ the value $\Psi_{c}$ different from its value in the midplane. This constant value $\Psi_{c}$ of $\Psi$ remains to be determined in the following. $Y(z)$ is then solution of:

$$
2 \lambda^{2} \Psi_{\mathrm{c}}^{2} Y_{z z z z}+4 \gamma(1-A) \Psi_{\mathrm{c}}^{2} Y_{z z}-\Psi_{\mathrm{c}}^{4}\left(Y_{, z}^{3}\right)_{, z}=0
$$

By integration, equation (C.7) leads to:

$$
2 \lambda^{2} \Psi_{\mathrm{c}}^{2} Y_{z z z}+4 \gamma(1-A) \Psi_{c}^{2} Y_{z}-\Psi_{\mathrm{c}}^{4} Y_{z}^{3}=\beta
$$

in which $Y(z)$ is a $\Lambda$-periodic function. Thus, the mean values over $\Lambda$ of $Y_{z z z}, Y_{, z}$ and $Y_{z z}^{3}$ equal zero and $\beta=0$. Equation (C.8) can be integrated again:

$$
\lambda^{2} \Psi_{\mathrm{c}}^{2} Y_{z z}^{2}+2 \gamma(1-A) \Psi_{\mathrm{c}}^{2} Y_{, z}^{2}-\frac{\Psi_{\mathrm{c}}^{4}}{4} Y_{z}^{4}=E_{z}
$$

in which $E_{z}$ is a constant. Setting

$$
\Psi_{c} Y_{, z}=\bar{\omega} p(s)
$$

with $s=z q_{z} / 2$, equation (C.9) becomes

$$
\frac{p_{, s}^{2}}{2}=\varepsilon_{z}-2 p^{2}+p^{4}
$$


with

$$
\begin{aligned}
q_{z} & =\frac{\sqrt{2 \gamma(1-A)}}{\lambda} \\
\bar{\omega} & =2 \sqrt{\gamma(1-A)}
\end{aligned}
$$

and $\varepsilon_{z}=E_{z} / \gamma^{2}(1-A)^{2}$. For convenience, we rewrite equation (C.11) in the form:

$$
\int_{0}^{p(s)} \frac{\mathrm{d} p}{\sqrt{\varepsilon_{z}-2 p^{2}+p^{4}}}=\sqrt{2} s
$$

where we have chosen $p=0$ for $s=0$ (i.e. $z=0$ ). One can see that $0 \leq p(s) \leq \sqrt{\zeta_{-}}$where

$$
\zeta_{--}=1-\sqrt{1-\varepsilon_{z}}
$$

is the smallest root of the equation $\varepsilon_{z}-2 p^{2}+p^{4}=0$ with $0 \leq \varepsilon_{z} \leq 1$. Denote that, for $p=\sqrt{\zeta_{-}}, s$ takes the maximal value

$$
\begin{aligned}
s_{\max } & =\frac{1}{\sqrt{2}} \int_{0}^{\sqrt{\zeta_{-}}} \frac{\mathrm{d} p}{\sqrt{\varepsilon_{z}-2 p^{2}+p^{4}}}=\frac{1}{\sqrt{2}} \int_{0}^{\sqrt{\zeta-}} \frac{\mathrm{d} p}{\sqrt{\left(\zeta_{+}-p^{2}\right)\left(\zeta_{-}-p^{2}\right)}} \\
& =\frac{1}{\sqrt{2 \zeta_{+}}} \int_{0}^{\pi / 2} \frac{\mathrm{d} t}{\sqrt{\left(1-\frac{\zeta_{-}}{\zeta_{+}} \sin ^{2} t\right)}}=\frac{1}{\sqrt{2 \zeta_{+}}} K\left(\frac{\zeta_{-}}{\zeta_{+}}\right)
\end{aligned}
$$

where $K$ is the complete elliptic integral of the first kind and $\zeta_{+}=1+\sqrt{1-\varepsilon_{z}}$. Finally, the shape of the undulation is determined by integrating $p(s)$ :

$$
\begin{aligned}
\Psi_{c} Y(z) & =\int_{0}^{z} \Psi_{c} Y_{, z}\left(z^{\prime}\right) \mathrm{d} z^{\prime}-C=\frac{2 \bar{\omega}}{q_{z}} \int_{0}^{z q_{z} / 2} p(s) \mathrm{d} s-C \\
& =\frac{\sqrt{2} \bar{\omega}}{q_{z}} \int_{0}^{\left.p\left(z q_{z} / 2\right)\right)} \frac{p \mathrm{~d} p}{\sqrt{\varepsilon_{z}-2 p^{2}+p^{4}}}-C \\
& =\frac{\sqrt{2} \bar{\omega}}{q_{z}} \operatorname{Ln}\left[\frac{\sqrt{\zeta_{+}-p^{2}}-\sqrt{\zeta_{-}-p^{2}}}{\sqrt{\zeta_{+}}-\sqrt{\zeta_{-}}}\right]-C
\end{aligned}
$$

where $C$ is a constant. Equations (C.15) and (C.17) give a parametric representation of the shape of the columns for $0 \leq z \leq \Lambda / 4$ (Fig. 5a). Indeed, for $s=0$ we have $p(0)=0$ and $p^{\prime}(0)=\sqrt{2 \varepsilon_{z}}$. At this point, $\frac{\partial v}{\partial z}=0$ and the curvature $\kappa$ is maximal and given by

$$
\kappa=\frac{\partial^{2} v}{\partial z^{2}}=\Psi Y_{, z z}=\bar{\omega} \frac{q_{z}}{2} p_{, s}=2 \Psi \gamma(1-A) \frac{\sqrt{\varepsilon_{z}}}{\lambda}
$$

For $s=s_{\max }$ we have $p\left(s_{\max }\right)=\sqrt{\zeta_{-}}$and $p^{\prime}\left(s_{\max }\right)=0$. Thus, the curvature of the columins equals zero and the inclination $\theta$ of the columns is maximal and given by

$$
\tan (\theta)=\frac{\partial v}{\partial z}=\Psi Y_{z}=2 \Psi \sqrt{\gamma(1-A) \zeta_{-}}
$$


The mean value of $Y(z)$ over the wavelength $\Lambda$ being equal to zero, $C$ equals the amplitude of $\Psi_{c} Y$ :

$$
C=\frac{\sqrt{2} \bar{\omega}}{q_{z}} \operatorname{Ln}\left[\frac{\sqrt{\zeta_{+}-\zeta_{-}}}{\sqrt{\zeta_{+}}-\sqrt{\zeta_{-}}}\right]
$$

The wavelength $\Lambda$ can be rewritten in the form

$$
\Lambda=\frac{8 s_{\max }}{q_{z}}=\frac{2}{q_{z}} \tau\left(\varepsilon_{z}\right)
$$

with

$$
\tau\left(\varepsilon_{z}\right)=\frac{2 \sqrt{2}}{\sqrt{\zeta_{+}}} K\left(\frac{\zeta_{-}}{\zeta_{+}}\right)
$$

Thus, $\varepsilon_{z}$ controls the shape of the columns since the maximal curvature and the maximal tilt angle $\theta$ of the columns increase with $\varepsilon_{z}$. When $\varepsilon_{z} \rightarrow 1$, the columns form a zigzag in the midplane. By contrast, when $\varepsilon_{z} \rightarrow 0$, they undulate following a sinusoidal law. Indeed, for small $\varepsilon_{z}$

$$
s=\frac{1}{\sqrt{2}} \int_{0}^{p(s)} \frac{\mathrm{d} p}{\sqrt{\varepsilon_{z}-2 p^{2}+p^{4}}} \approx \frac{1}{2} \int_{0}^{\sqrt{\frac{2}{\varepsilon_{z}}} p(s)} \frac{\mathrm{d} \phi}{\sqrt{1-\phi^{2}}}=\frac{1}{2} \sin ^{-1}\left(\sqrt{\frac{2}{\varepsilon_{z}}} p(s)\right)
$$

and

$$
p(s) \approx \sqrt{\frac{\varepsilon_{z}}{2}} \sin (2 s) \quad\left(\varepsilon_{z} \rightarrow 0\right)
$$

In conclusion, we have determined the solution $Y(z)$ to the differential equation (C.6a) in which we have neglected the term $2 \bar{A} \Psi_{, x}^{2} Y$. Nevertheless, it is possible to take approximately into account this previously neglected term by correcting the undulation wavelength. Indeed, assuming $\Psi Y$ is a periodic function of wavelength $\frac{4 \pi}{q_{z}}, \Psi Y=w\left(\imath \frac{z q_{z}}{2}\right)$, we obtain after substitution in equation (C.6a):

$$
\left(2 \lambda^{2}-\vartheta^{2}\right)\left(\frac{q_{z}}{2}\right)^{4}-4 \gamma(1-A)\left(\frac{q_{z}}{2}\right)^{2}+\frac{2 \pi^{2} \bar{A}}{d^{2}}=0
$$

where $\vartheta$ is a length related to the shape and to the amplitude of $w$. The last term in equation (C.24) was previously neglected. To calculate $\vartheta$, we neglect this term in equation (C.24) and we take for $q_{z}$ its value given by equation (C.12). It yields

$$
q_{z}=\frac{\sqrt{2 \gamma(1-A)}}{\lambda}=4 \sqrt{\frac{\gamma(1-A)}{2 \lambda^{2}-\vartheta^{2}}}
$$

This relation gives the unknown quantity $2 \lambda^{2}-\vartheta^{2}$. Then, we replace $2 \lambda^{2}-\vartheta^{2}$ by its value in equation (C.24) and we solve it in $q_{z}$. It gives:

$$
q_{z}=\frac{\sqrt{\gamma(1-A)}}{\lambda}\left(1+\sqrt{1-\left(\frac{\gamma_{c}}{\gamma}\right)^{2}}\right)^{1 / 2}
$$

We will use this expression of $q_{z}$ in the following computations. This correction to $q_{z}$ allows us to find again the threshold of instability and to describe approximately the solution in the weakly nonlinear regime. 


\section{Amplitude Modulation $\Psi(x)$ in the Thickness}

An approximate solution $\Psi(x)$ can be found by solving equation (C.6b) in which $Y(z)$ and its derivatives are replaced by their mean values over the wavelength:

$$
2 \bar{A}<Y^{2}>_{\Lambda} \Psi_{x x}+\left[4 \gamma(1-A)<Y_{, z}^{2}>_{\Lambda}-2 \lambda^{2}<Y_{, z z}^{2}>_{\Lambda}\right] \Psi-<Y_{, z}^{4}>_{\Lambda} \Psi^{3}=0
$$

Its integration gives

$$
\begin{aligned}
& \frac{1}{2}\left[2 \bar{A}<\Psi_{\mathrm{c}}^{2} Y^{2}>_{\Lambda}\right]\left(\frac{\Psi_{, x}}{\Psi_{\mathrm{c}}}\right)^{2}+\frac{1}{2}\left[4 \gamma(1-A)<\Psi_{\mathrm{c}}^{2} Y_{z}^{2}>_{\Lambda}-2 \lambda^{2}<\Psi_{\mathrm{c}}^{2} Y_{z z}^{2}>_{\Lambda}\right]\left(\frac{\Psi}{\Psi_{\mathrm{c}}}\right)^{2} \\
& -\left[\frac{1}{4}<\Psi_{\mathrm{c}}^{4} Y_{z}^{4}>_{\Lambda}\right]\left(\frac{\Psi}{\Psi_{\mathrm{c}}}\right)^{4}=E_{x}
\end{aligned}
$$

where $E_{x}$ is a constant. In the following, we set

$$
\frac{\Psi(x)}{\Psi_{\mathrm{c}}}=\nu f(t) \text { with } t=\frac{x q}{2}
$$

Equation (C.28) then rewrites in the form

$$
\frac{f_{t,}^{2}}{2}=\varepsilon_{x}-2 f^{2}+f^{4}
$$

with

$$
\nu=\frac{\sqrt{\gamma(1-A)}}{\bar{\omega}} \sqrt{\frac{\left\langle p^{2}>_{\Lambda}-\frac{1}{4} \eta<p_{, s}^{2}>_{\Lambda}\right.}{<p^{4}>_{\Lambda}}}
$$

and

$$
q=\frac{\tau\left(\varepsilon_{x}\right)}{\mathrm{d}}=q_{z} \sqrt{\frac{\gamma(1-A)}{2 \bar{A}}} \sqrt{\frac{\left\langle p^{2}>_{\Lambda}-\frac{1}{4} \eta\left\langle p_{, s}^{2}>_{\Lambda}\right.\right.}{<\left(\int p \mathrm{~d} s\right)^{2}>_{\Lambda}}}
$$

with $\eta=\lambda^{2} q_{z}^{2} / 2 \gamma(1-A)$ and $\varepsilon_{x}$ a constant. Indeed, in the midplane, $\Psi_{, x}=0$ so that $q=\tau\left(\varepsilon_{x}\right) / d$. The mean values are defined as follows:

$$
\begin{aligned}
<p^{2}>_{\Lambda} & =\frac{1}{\bar{\omega}^{2}}<\left(\Psi_{c} Y_{z}\right)^{2}>_{\Lambda}=\frac{4}{\Lambda} \int_{0}^{\Lambda / 4} p^{2} \mathrm{~d} z \\
& =\frac{1}{\tau\left(\varepsilon_{z}\right)} \int_{0}^{\tau\left(\varepsilon_{z}\right)} p^{2} \mathrm{~d} s=\frac{\pi \zeta_{-}}{\tau\left(\varepsilon_{z}\right) \sqrt{2 \zeta_{+}}}{ }_{2} F_{1}\left[\frac{1}{2}, \frac{3}{2}, 2, \frac{\zeta_{-}}{\zeta^{+}}\right] \\
<p^{4}>_{\Lambda} & =\frac{1}{\overline{\bar{\omega}}^{4}}<\left(\Psi_{\mathrm{c}} Y_{z}\right)^{4}>_{\Lambda}=\frac{3 \pi \zeta_{-}^{2}}{4 \tau\left(\varepsilon_{z}\right) \sqrt{2 \zeta_{+}}}{ }_{2} F_{1}\left[\frac{1}{2}, \frac{5}{2}, 3, \frac{\zeta_{-}}{\zeta_{+}}\right] \\
<p_{, s}^{2}>_{\Lambda} & =\frac{4}{q_{z}^{2} \bar{\omega}^{2}}\left\langle\left(\Psi_{\mathrm{c}} Y_{, z z}\right)^{2}>_{\Lambda}=\frac{\pi \zeta_{-} \sqrt{2 \zeta_{+}}}{\tau\left(\varepsilon_{z}\right)}{ }_{2} F_{1}\left[\frac{-1}{2}, \frac{1}{2}, 2, \frac{\zeta_{-}}{\zeta_{+}}\right]\right.
\end{aligned}
$$




$$
\begin{aligned}
<\left(\int p \mathrm{~d} s\right)^{2}>_{\Lambda} & =\frac{q_{z}^{2}}{4 \bar{\omega}^{2}}<\left(\Psi_{c} Y\right)^{2}>_{\Lambda} \\
& =\frac{\sqrt{2}}{\tau\left(\varepsilon_{z}\right) \sqrt{\zeta_{+}}} \int_{0}^{\pi / 2} \frac{\left(\operatorname{Ln}\left[\frac{\sqrt{\zeta_{+}-\zeta_{-} \sin ^{2}[\theta]}-\sqrt{\zeta_{-}} \cos [\theta]}{\sqrt{\zeta_{+}-\zeta_{-}}}\right]\right)^{2}}{\sqrt{1-\frac{\zeta_{-}}{\zeta_{+}} \sin ^{2}[\theta]}} \mathrm{d} \theta
\end{aligned}
$$

where ${ }_{2} F_{1}$ is the hypergeometric function. The last mean value (Eq. (C.33d)) must be calculated numerically. Equation (C.28) expresses the coupling between the undulation and the finite-thickness effects: for a given value of $\varepsilon_{z}$ (i.e. for a given value of the wavelength $\Lambda$ (Eq. (3.21))), one can determine $\varepsilon_{x}$ (i.e. the shape of the amplitude $\Psi(x)$ within the sample thickness). For small $\varepsilon_{x}, \Psi(x)$ varies like a sinus whereas it resembles a trapezoid as $\varepsilon_{x}$ is close to 1 (Fig. 5). The amplitude of the undulation vanishes close to the limiting glass plates over a characteristic length $T d / 2$ where $T$ is given by:

$$
T=\frac{4}{\tau\left(\varepsilon_{x}\right)} \sqrt{\frac{\zeta_{-}^{*}}{2 \varepsilon_{x}}}
$$

where $\zeta_{-}^{*}=1-\sqrt{1-\varepsilon_{x}}$.

\section{Density of Free Energy, Minimization and Asymptotic Behaviors}

For known functions $Y(z)$ and $\Psi(x)$, the mean density of free energy over the wavelength and the thickness reads:

$$
\ll \tilde{f}>_{\Lambda}>_{d}=4 \gamma^{2}(1-A)+4 \gamma^{2}(1-A)^{2} \frac{\left(<p^{2}>_{\Lambda}-\frac{1}{4} \eta<p_{, s}^{2}>_{\Lambda}\right)^{2}}{<p^{4}>_{\Lambda}}\left(<f_{, t}^{2}>_{d}-\varepsilon_{x}\right)
$$

where the definition of $\left\langle f_{, t}^{2}\right\rangle_{d}$ is similar to the definition of $\left\langle p_{, s}^{2}\right\rangle_{\Lambda}$. For a given value of $\Lambda$, equation (C.21) gives $\varepsilon_{z}$, which allows us to calculate the mean values over the wavelength of $Y$ and of its derivatives using equations (C.33a-33d). The next step consists of determining $\varepsilon_{x}$ from equation (C.32) and the mean density of free energy from equation (C.35).

At last, we must minimize $\left.\ll \tilde{f}>_{\Lambda}\right\rangle_{d}$ with respect to $\Lambda$. This has been done numerically (see Figs. 5 and 6 in Sect. 4). Nevertheless, asymptotic behaviors for $\gamma \approx \gamma_{c}$ and $\gamma \gg \gamma_{c}$ can be found analytically:

If $\gamma \approx \gamma_{c}$, then $\varepsilon_{x}$ and $\varepsilon_{z}$ vanish. In this limit, $<p^{2}>_{\Lambda} \approx \varepsilon_{z} / 4,<p_{, s}^{2}>_{\Lambda} \approx \varepsilon_{z}$, $<\left(\int p \mathrm{~d} s\right)^{2}>_{\Lambda} \approx \varepsilon_{z} / 16, \tau\left(\varepsilon_{x}\right) \approx \pi / 2$ and $\tau\left(\varepsilon_{z}\right) \approx \pi / 2$. Substitution in equations (C.21) and (C.32) gives again the threshold of instability $\gamma_{c}$ and the corresponding critical wavelength $\Lambda_{c}$.

If $\gamma \gg \gamma_{c}$, then $\varepsilon_{\mathrm{x}}$ and $\varepsilon_{z}$ tend to 1 . In this limit, $<p^{2}>_{\Lambda} \approx 1-\frac{2 \sqrt{2}}{\tau\left(\varepsilon_{z}\right)},<p_{, s}^{2}>_{\Lambda} \approx$ $1-\frac{8 \sqrt{2}}{3 \tau\left(\varepsilon_{z}\right)},<p^{4}>_{\Lambda} \approx \frac{8 \sqrt{2}}{3 \tau\left(\varepsilon_{z}\right)}$ and $<f_{, t}^{2}>_{\Lambda} \approx 1-\frac{8 \sqrt{2}}{3 \tau\left(\varepsilon_{x}\right)}$. As the columns form a zigzag, we find geometrically that $\left\langle\left(\int p \mathrm{~d} s\right)^{2}>_{\Lambda} \approx \frac{\tau\left(\varepsilon_{z}\right)}{48}\right.$ Then, introducing these expressions in 
equation (C.35), it is easy to show that the wavelength is given by:

$$
\Lambda=\left(\frac{2 \sqrt{3}}{\pi}\right)^{1 / 2} \Lambda_{\mathrm{c}} .
$$

The maximal curvature $\kappa$ and the maximal tilt angle $\theta$ of the columns are given by equation (C.18) and (C.19) with $\varepsilon_{x}=1$ and $\varepsilon_{z}=1$. The relative thickness of the twist boundary layer $\chi=\frac{\pi}{2} T$ over which the amplitude of the undulation vanishes is then given by:

$$
\chi=\left(\frac{\pi}{2 \sqrt{3}}\right)^{1 / 2} \sqrt{\frac{\gamma_{c}}{\gamma}} .
$$

\section{References}

[1] Gilchrist C.A., Rogers J., Steel G., Vaal E.G., Winsor P.A., J. Colloïd Interface Sci. 25 (1967) 409-420.

[2] Rogers J., Winsor P.A., J. Collö̈d Interface Sci. 30 (1969) 500.

[3] a) Livolant F., Bouligand F., J. Phys. France 47 (1986) 1813; b) Livolant F., Leforestier A., Mol. Cryst. Liq. Cryst. 215 (1992) 47; c) Durand D., Doucet J., Livolant F., J. Phys. II France 2 (1992) 1769.

[4] Gharbia M., Cagnon M., Durand G., J. Phys. Lett. France 46 (1985) L-683.

[5] Kléman M., Oswald P., J. Phys. France 43 (1982) 655.

[6] Cagnon M., Gharbia M., Durand G., Phys. Rev. Lett. 53 (1984) 938.

[7] Prost J., Liq. Cryst. 8 (1990) 123.

[8] Gharbia M., Othman T., Gharbi A., Destrade C., Durand G., Phys. Rev. Lett. 68 (1992) 2031.

[9] Palierne J.F., Thèse de 3ème Cycle, Université de Paris-Sud (1983).

[10] Sallen L., Oswald P., Géminard J.C., Malthête J., J. Phys. II France 5 (1995) 937.

[11] a) Clerc M., Thesis, Université de Paris Sud, Centre d'Orsay (1992); b) Davidson P., Clerc M., Ghosh S.S., Maliszewkyj N.C., Heiney P.A., Hynes J., Smith A.B. III, preprint.

[12] Mitchell D.J., Tiddy G.J.T., Waring L., Bostock T., McDonald M.P., J. Chem. Phys. Faraday Trans.1 79 (1983) 975.

[13] Clunie J.S., Goodman J.F., Symons P.C., Trans. Faraday Soc., 65 (1969) 287.

[14] Petrov A.G., Durand G., J. Phys. Lett. France 44 (1983) L793.

[15] Oswald P., Moulin M., Metz P., Géminard J.C., Sotta P., Sallen L., J. Phys. III France 3 (1993) 1891.

[16] Clerc M., private communiction. This estimation of the elastic anisotropy $A$ was obtained from the analysis in terms of thermal phonons of the diffuse scattering observed about the 6 Bragg peaks in a plane perpendicular to the cylinders.

[17] Singer S.J., Phys. Rev. E 48 (1993) 2796.

[18] Sornette D., J. Phys. France 48 (1987) 151.

[19] Oswald P., Kléman M., J. Phys. France 42 (1981) 1461; Oswald P., Thèse de 3ème Cycle, Université de Paris Sud, Centre d'Orsay, 1981.

[20] Ribière P., Pirkl S., Oswald P., Liq. Cryst. 16 (1994) 203. 